\title{
Comparison of Long-Term Clinical Outcomes Between Sirolimus-Eluting Stents and Paclitaxel-Eluting Stents Following Rotational Atherectomy
}

\author{
Ryo Naito, ${ }^{1}$ MD, Kenichi SaKakura, ${ }^{1}$ MD, Hiroshi Wada, ${ }^{1}$ MD, Hiroshi Funayama, ${ }^{1}$ MD, \\ Yoshitaka Sugawara, ${ }^{1}$ MD, Norifumi Kubo, ${ }^{1}$ MD, Junya AKo, ${ }^{1}$ MD, and Shin-ichi Momomura, ${ }^{1}$ MD
}

\begin{abstract}
SUMMARY
Rotational atherectomy (RA) can facilitate smooth stent delivery and stent expansion through lesion modification for a calcified coronary lesion. Several studies reported that sirolimus-eluting stent (SES) implantation following RA showed a lower rate of revascularization compared with bare-metal stents (BMS). However, there are limited data that compared the clinical outcomes between SES and paclitaxel-eluting stents (PES) after RA. We compared the long-term clinical outcomes of SES and PES following RA. Two hundred and thirty-three consecutive patients (SES $n=179$, PES $n=54$ ) who were treated with SES or PES following RA between $10^{\text {th }}$ September 2004 and $13^{\text {th }}$ April 2010 were investigated. Follow-up data for clinical outcomes were obtained in $91.4 \%$ of all subjects. The median follow-up period was 630 days (interquartile range, 300 to 1170 days) in the SES group, and 625 days (interquartile range, 285 to 900 days) in the PES group. Clinical outcomes including target lesion revascularization (TLR) (SES 4.9\% versus PES 9.8\%, $P=$ 0.31 ), target vessel revascularization (TVR) (SES 6.8\% versus PES 11.8\%, $P=0.25$ ), and major adverse cardiac events (MACE) (SES $14.8 \%$ versus PES $13.7 \%, P=0.8$ ) were not statistically different between the groups. The unadjusted cumulative event rates estimated by the Kaplan-Meier method and the log-rank test showed no significant differences between the two groups for time to event for TLR, cardiovascular death, all-cause death, or MACE. In conclusion, there was no significant difference in the long-term clinical outcomes between SES and PES following RA. (Int Heart J
\end{abstract} 2012; 53: 149-153)

Key words: Sirolimus-eluting stents, Paclitaxel-eluting stents, Rotational atherectomy, Calcified coronary lesion

$\mathrm{C}$ alcified coronary lesions and diffuse long lesions are still one of the challenging coronary artery lesion subsets, despite the advances in percutaneous coronary artery intervention (PCI) devices and techniques. ${ }^{1-3)}$ Rotational atherectomy (RA) has emerged to be a representative device for such complex lesions. RA can facilitate smooth stent delivery and stent expansion through lesion modification. ${ }^{4 \cdot 6}$ However, the clinical outcomes after implanting a baremetal stent (BMS) following RA have not been satisfactory. ${ }^{7-9)}$

Drug-eluting stents (DES) have dramatically reduced the rate of revascularization compared with BMS. ${ }^{10-13)}$ With regard to calcified lesions, several studies reported that sirolimus-eluting stent (SES) implantation following RA had a lower rate of revascularization than BMS. ${ }^{14,15}$ Other studies demonstrated that long-term clinical outcomes of DES implantation after RA were feasible. ${ }^{16,17)}$ In these studies, multiple types of DES including SES and paclitaxel-eluting stents (PES) were implanted. However, data that compared the clinical outcomes between SES and PES after RA are limited. Therefore, we compared the long-term clinical outcomes of SES and PES following RA.

\begin{abstract}
Methods
Patients: We identified patients from our PCI database who received SES or PES implantation following rotational atherectomy (RA) between $10^{\text {th }}$ September 2004 and $13^{\text {th }}$ April 2010. Follow-up data were obtained until $5^{\text {th }}$ September 2011. Follow-up coronary angiography (CAG) was defined as a first CAG during chronic phase (6 to 18 months) after the initial procedure. When a patient was treated with both SES and PES in two or more sessions, only the initial procedure was included. We excluded patients who were treated with both SES and PES simultaneously.

Definitions: Angiographic success was defined as residual instent stenosis of $<30 \%$ with Thrombolysis in Myocardial Infarction (TIMI) 3 flow at the end of the procedure. Procedurerelated adverse events comprised coronary dissection, coronary perforation, non-Q wave myocardial infarction (MI), and Q wave MI. Major adverse cardiac events (MACE) included target vessel revascularization, coronary artery bypass graft, nonfatal MI, cardiovascular death, and all-cause death. MI was defined as CK elevation greater than two times the normal levels. Stent thrombosis (ST) was assessed according to Academic Research Consortium definitions. ${ }^{18)}$
\end{abstract}

From the ${ }^{1}$ Division of Cardiology, Department of Integrated Medicine I, Saitama Medical Center, Jichi Medical University School of Medicine, Saitama, Japan. Address for correspondence: Kenichi Sakakura, MD, 847-1, Amanuma-cho, Oomiya-ku, Saitama, Saitama 330-8503, Japan.

Received for publication December 5, 2011.

Revised and accepted March 5, 2012. 
Other clinical criteria were defined as follows: hypertension was defined as systolic blood pressure $\geq 140 \mathrm{mmHg}$, diastolic blood pressure $\geq 90 \mathrm{mmHg}$, or medical treatment for hypertension. Diabetes mellitus was defined as hemoglobin A1c $\geq 6.5 \%$ or treatment for diabetes mellitus. Dyslipidemia was defined as a total cholesterol level $\geq 220 \mathrm{mg} / \mathrm{dL}$, low-density lipoprotein cholesterol level $\geq 140 \mathrm{mg} / \mathrm{dL}$, high-density lipoprotein cholesterol level $<40 \mathrm{mg} / \mathrm{dL}$, or treatment for dyslipidemia. Reference diameter and lesion length were calculated by quantitative coronary angiographic analysis using off line, computer-based software (QAngio XA ver.7.1, MEDIS Imaging Systems, Leiden, Netherlands). The burr-to-artery ratio was defined as the final burr size divided by the reference diameter. This study was approved by the Institutional Review Board of Jichi Medical University Saitama Medical Center.

Rotational atherectomy and stent implantation: The indications for rotational atherectomy (RA) in our institution were as follows: (i) moderate or severe calcified lesions, (ii) diffuse lesions which were expected to be difficult to be treated with stenting alone, and (iii) ostial lesions. When treating the culprit lesions in acute coronary syndrome (ACS), RA was not used as first-line therapy. However, RA was used for the culprit lesion in ACS, if necessary. RA was performed using standard techniques. In brief, all patients received oral aspirin (at least $81 \mathrm{mg}$ ) before the procedure. Ticlopidine (200 mg) or clopidogrel $(75 \mathrm{mg}$ ) was added when coronary stenting was performed. Intravenous heparin was used to achieve an appropriate activated coagulation time ( $\geq 250$ seconds). The lesion was crossed with a 0.009 inch guide wire. The rotablator burr was subsequently advanced over the wire to a position proximal to the lesion. The burr was activated and moved with a pecking motion. Individual burring runs were performed at a speed of 180,000 - 220,000 rpm while being careful to avoid a decrease $\geq 5,000 \mathrm{rpm}$. Intracoronary verapamil $(5 \mathrm{mg}$ ) or nicorandil (12 $\mathrm{mg})$, isosorbide dinitrate $(2.5 \mathrm{mg})$, heparin $(10,000$ units $)$, and normal saline $(500 \mathrm{~mL})$ were routinely used as drug cocktail for preventing slow flow.

Statistical analysis: Data for continuous variables are ex-

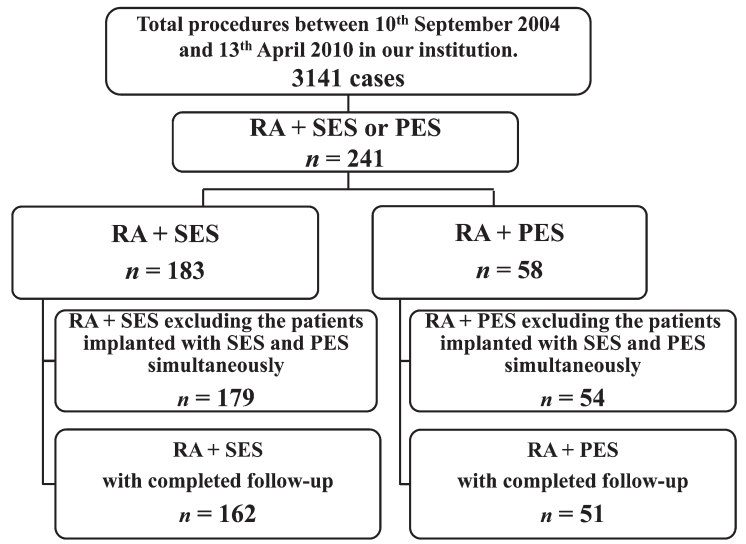

Figure 1. Flow chart of study population. Study population was identified from our percutaneous coronary intervention (PCI) database between $10^{\text {th }}$ September 2004 and $13^{\text {th }}$ April 2010. SES indicates sirolimus-eluting stents and PES, paclitaxel-eluting stents. pressed as the mean \pm SD and categorical variables are expressed as frequencies. Continuous variables were compared using the unpaired $t$-test or Mann-Whitney $U$ test, and categorical variables using the chi-square test or Fischer's exact test. $P$ values $<0.05$ were considered as significant. Unadjusted cumulative event rates were estimated by the Kaplan-Meier method and compared with the log-rank test. All statistical analyses were performed using SPSS version 18.0 statistical software (IBM SPSS Inc, Chicago, IL, USA).

\section{RESULTS}

DES implantation following RA for angiographically calcified coronary stenosis was performed in 241 consecutive patients between $10^{\text {th }}$ September 2004 and $13^{\text {th }}$ April 2010. Among the 241 patients, 183 were treated with sirolimus-eluting stents $\left(\right.$ Cypher ${ }^{\circledR}$, Cordis, Johnson \& Johnson Corporation, Miami Lakes, FL) and 58 were treated with paclitaxel-eluting stents (Taxus Express or Taxus Liberté, Boston Scientific Corporation, Natick, MA). PES have been used since $26^{\text {th }}$ June, 2007. Stent selection was left to the discretion of the operators. Of the 241 patients, 8 were excluded because SES and PES were simultaneously implanted in the identical procedure. The remaining 233 patients were investigated (Figure 1) (SES; $n=$ 179, PES; $n=54)$. In 233 patients, $143(61.4 \%)$ underwent follow-up CAG (SES; $n=114$ (63.7\%), PES; $n=29(53.7 \%)$ ).

The baseline characteristics of the patients are shown in

Table I. Baseline Characteristics of SES Group and PES Group

\begin{tabular}{lccc}
\hline & SES & PES & \\
& $(n=179)$ & $(n=54)$ & $P$ \\
\hline Age, years & $69.0 \pm 8.8$ & $67.3 \pm 9.3$ & 0.20 \\
Male sex, $n(\%)$ & $133(74.3)$ & $43(79.6)$ & 0.43 \\
Body mass index, kg/m ${ }^{2}$ & $23.7 \pm 3.1$ & $23.4 \pm 3.0$ & 0.47 \\
Hypertension, $n(\%)$ & $130(72.6)$ & $42(77.7)$ & 0.45 \\
Diabetes mellitus, $n(\%)$ & $90(50.3)$ & $23(42.6)$ & 0.32 \\
Dyslipidemia, $n(\%)$ & $129(72.1)$ & $43(79.6)$ & 0.27 \\
Current smoking, $n(\%)$ & $31(17.3)$ & $13(24.1)$ & 0.27 \\
Family history, $n(\%)$ & $54 / 172(31.4)$ & $15 / 52(28.8)$ & 0.73 \\
Hemodialysis, $n(\%)$ & $22(12.3)$ & $7(13.0)$ & 0.90 \\
Medical treatments, $n(\%)$ & & & \\
Aspirin & $176(98.3)$ & $58(100)$ & 1.0 \\
Clopidogrel & $\underline{72(40.2)}$ & $41(75.9)$ & $\leq 0.001$ \\
Ticlopidine & $101(56.4)$ & $\underline{13(24.1)}$ & $\leq 0.001$ \\
Cirostazol & $5(2.8)$ & $1(1.9)$ & 1.0 \\
Statin & $143(79.9)$ & $48(88.9)$ & 0.13 \\
Beta blocker & $82(45.8)$ & $26(48.1)$ & 0.76 \\
Angiotensin receptor blocker & $67(37.4)$ & $23(42.6)$ & 0.50 \\
Angiotension converting en- & $56(31.3)$ & $20(37.0)$ & 0.43 \\
$\quad$ zyme inhibitor & & & \\
Calcium channel blocker & $76(42.5)$ & $15(27.8)$ & 0.05 \\
Oral hypoglycemic agents & $44(24.6)$ & $12(22.2)$ & 0.72 \\
Insulin & $36(20.1)$ & $6(11.1)$ & 0.13 \\
Clinical presentation, $n(\%)$ & & & \\
Acute myocardial infarction & $6(3.4)$ & $5(9.3)$ & 0.13 \\
Unstable angina pectoris & $13(7.3)$ & $4(7.4)$ & 1.0 \\
Acute coronary syndrome & $19(10.6)$ & $9(16.7)$ & 0.23 \\
History of myocardial & $48(26.8)$ & $21(38.9)$ & 0.09 \\
$\quad$ infarction & & & \\
Chronic total occlusion & $22(12.3)$ & $7(13.0)$ & 1.0 \\
\hline
\end{tabular}

Values are mean \pm SD or numbers of patients (percentages). 
Table II. Procedural Characteristics of SES Group and PES Group

\begin{tabular}{|c|c|c|c|}
\hline & $\begin{array}{c}\text { SES } \\
(n=179)\end{array}$ & $\begin{array}{c}\text { PES } \\
(n=54)\end{array}$ & $P$ \\
\hline Stent number & $1.54 \pm 0.7$ & $1.56 \pm 0.7$ & 0.90 \\
\hline Stent diameter, $\mathrm{mm}$ & $\underline{2.84 \pm 0.3}$ & $\underline{2.94 \pm 0.3}$ & $\underline{0.04}$ \\
\hline Stent length, mm & $35.6 \pm 17.5$ & $35.0 \pm 17.3$ & 0.86 \\
\hline Pressure of stent dilatation, atm & $\underline{19.3 \pm 2.6}$ & $\underline{15.6 \pm 3.1}$ & $\leq 0.001$ \\
\hline \multicolumn{3}{|l|}{ AHA classification } & 0.13 \\
\hline \multicolumn{4}{|l|}{ Target lesion, $n(\%)$} \\
\hline $\begin{array}{l}\text { Left anterior descending } \\
\text { artery }\end{array}$ & $132(73.7)$ & $31(57.4)$ & $\underline{0.03}$ \\
\hline Left circumflex & $19(10.6)$ & $6(11.1)$ & \\
\hline Right coronary artery & $28(15.6)$ & $17(31.5)$ & \\
\hline Bifurcation of left main trunk & $25(14)$ & $11(20.4)$ & 0.25 \\
\hline Procedural success, $n(\%)$ & $179(100)$ & $58(100)$ & 0.76 \\
\hline Reference diameter, $\mathrm{mm}$ & $2.2 \pm 0.5$ & $2.3 \pm 0.5$ & 0.05 \\
\hline Lesion length, $\mathrm{mm}$ & $\underline{22.0 \pm 12.9}$ & $\underline{18.9 \pm 12.8}$ & $\underline{0.04}$ \\
\hline Burr size & $1.65 \pm 0.2$ & $1.64 \pm 0.2$ & 0.73 \\
\hline Burr-to-artery ratio & $0.8 \pm 0.3$ & $0.7 \pm 0.1$ & 0.06 \\
\hline Burr-to-artery ratio $>0.7, n(\%)$ & $122(68.2)$ & $35(60.3)$ & 0.23 \\
\hline Complications, $n(\%)$ & $6(3.4)$ & $2(3.7)$ & 1.0 \\
\hline Coronary dissection, $n(\%)$ & $4(2.2)$ & 0 & 0.58 \\
\hline Coronary perforation, $n(\%)$ & $1(0.6)$ & 0 & 1.0 \\
\hline Non Q wave MI, $n(\%)$ & $1(0.6)$ & $2(3.7)$ & 0.14 \\
\hline Q wave MI, $n(\%)$ & 0 & 0 & \\
\hline $\begin{array}{l}\text { CAG during chronic phase, } n \\
\quad(\%)\end{array}$ & $114(63.7)$ & $29(53.7)$ & 0.16 \\
\hline Clinical follow-up rate, $n(\%)$ & $162(90.5)$ & $51(94.4)$ & 0.42 \\
\hline
\end{tabular}

Values are mean \pm SD or numbers of patients (percentages). MI indicates myocardial infarction.

Table I. The only significant differences between the groups were for ticlopidine and clopidogrel use. Procedural characteristics are shown in Table II. With respect to lesion location, there were significantly more patients with a left anterior descending artery lesion in the SES group $(P=0.03)$. Lesion length was longer in the SES group $(P=0.04)$. Stent diameter was smaller and the pressure of stent dilatation was higher in the SES group $(P=0.04$ and $<0.001$, respectively). There was no significant difference in the RA procedure between the two groups (Table II).

Procedural success and procedure-related adverse events: Procedural success was achieved in all patients. Procedure-related adverse events were not statistically different between the groups (SES; 3.4\% and PES; 3.7\%, $P=1.0$ ) (Table II).

Clinical outcomes: Follow-up data for clinical outcomes were obtained in $91.4 \%$ of all subjects (SES; $90.5 \%$ and PES; $94.4 \%$ ). The median follow-up period was 630 days (interquartile range, 300 to 1170 days) in the SES group, and 625 days (interquartile range, 285 to 900 days) in the PES group. Clinical outcomes were not statistically different between the groups. The rate of TLR was $4.9 \%$ in the SES group and $9.8 \%$ in the PES group $(P=0.31)$. MACE occurred in $14.8 \%$ of the SES group and $13.7 \%$ of the PES group $(P=0.8)$. ST was identified in only the SES group $(n=3,1.9 \%)$ (Table III). ST occurred in two patients within the first 30 days after the procedure and the remaining one event occurred within 60 days after the procedure. The unadjusted cumulative event rates estimated by the Kaplan-Meier method and the log-rank test
Table IIIa. Clinical Outcomes of All Patients

\begin{tabular}{lccc}
\hline & $\begin{array}{c}\text { SES } \\
(n=162)\end{array}$ & $\begin{array}{c}\text { PES } \\
(n=51)\end{array}$ & $P$ \\
\hline TLR, $n(\%)$ & $8(4.9)$ & $5(9.8)$ & 0.31 \\
TVR, $n(\%)$ & $11(6.8)$ & $6(11.8)$ & 0.25 \\
CABG, $n(\%)$ & $9(5.6)$ & 0 & 0.12 \\
Nonfatal MI, $n(\%)$ & $4(2.5)$ & $1(2.0)$ & 1.0 \\
Stent thrombosis, $n(\%)$ & $3(1.9)$ & 0 & 1.0 \\
Hospitalization for angina pectoris, $n(\%)$ & $33(20.4)$ & $9(17.6)$ & 0.67 \\
Hospitalization for heart failure, $n(\%)$ & $8(4.9)$ & $4(7.8)$ & 0.5 \\
Cardiovascular death, $n(\%)$ & $3(1.9)$ & $1(2.0)$ & 1.0 \\
All death, $n(\%)$ & $12(7.4)$ & $2(3.9)$ & 0.53 \\
MACE, $n(\%)$ & $24(14.8)$ & $7(13.7)$ & 0.8 \\
\hline
\end{tabular}

Values are numbers of patients (percentages). TLR indicates target lesion revascularization; TVR, target vessel revascularization; CABG, coronary artery bypass graft; nonfatal MI, non-fatal myocardial infarction; and MACE, major adverse cardiac events.

Table IIIb. Clinical Outcomes of Non-Hemodialysis Patients

\begin{tabular}{lccc}
\hline & $\begin{array}{c}\text { SES } \\
(n=142)\end{array}$ & $\begin{array}{c}\text { PES } \\
(n=45)\end{array}$ & $P$ \\
\hline TLR, $n(\%)$ & $7(4.9)$ & $4(8.9)$ & 0.26 \\
TVR, $n(\%)$ & $10(7.0)$ & $5(11.1)$ & 0.28 \\
CABG, $n(\%)$ & $9(6.3)$ & 0 & 0.08 \\
Nonfatal MI, $n(\%)$ & $2(1.4)$ & $1(2.2)$ & 0.56 \\
Stent thrombosis, $n(\%)$ & $2(1.4)$ & 0 & 0.58 \\
Hospitalization for angina pectoris, $n(\%)$ & $28(19.7)$ & $6(13.3)$ & 0.33 \\
Hospitalization for heart failure, $n(\%)$ & $5(3.5)$ & $4(8.9)$ & 0.14 \\
Cardiovascular death, $n(\%)$ & $1(0.7)$ & $1(2.2)$ & 0.42 \\
All death, $n(\%)$ & $6(4.2)$ & $1(2.2)$ & 0.47 \\
MACE, $n(\%)$ & $22(15.5)$ & $6(13.3)$ & 0.72 \\
\hline
\end{tabular}

Values are numbers of patients (percentages). TLR indicates target lesion revascularization; TVR, target vessel revascularization; $\mathrm{CABG}$, coronary artery bypass graft; nonfatal MI, nonfatal myocardial infarction; and MACE, major adverse cardiac events.

Table IIIc. Clinical Outcomes of Hemodialysis Patients

\begin{tabular}{lccc}
\hline & $\begin{array}{c}\text { SES } \\
(n=20)\end{array}$ & $\begin{array}{c}\text { PES } \\
(n=6)\end{array}$ & $P$ \\
\hline TLR, $n(\%)$ & $1(5)$ & $1(16.7)$ & 0.42 \\
TVR, $n(\%)$ & $1(5)$ & $1(16.7)$ & 0.42 \\
CABG, $n(\%)$ & 0 & 0 & \\
Nonfatal MI, $n(\%)$ & $2(10)$ & 0 & 0.59 \\
Stent thrombosis, $n(\%)$ & $1(5)$ & 0 & 0.77 \\
Hospitalization for angina pectoris, $n(\%)$ & $5(25)$ & $3(50)$ & 0.25 \\
Hospitalization for heart failure, $n(\%)$ & $3(15)$ & 0 & 0.44 \\
Cardiovascular death, $n(\%)$ & $2(10)$ & 0 & 0.59 \\
All death, $n(\%)$ & $6(30)$ & $1(16.7)$ & 0.47 \\
MACE, $n(\%)$ & $8(40)$ & $1(16.7)$ & 0.3 \\
\hline
\end{tabular}

Values are numbers of patients (percentages). TLR indicates target lesion revascularization; TVR, target vessel revascularization; CABG, coronary artery bypass graft; nonfatal MI, nonfatal myocardial infarction; and MACE, major adverse cardiac events.

showed no significant difference between the two groups for time to event for TLR, cardiovascular death, all-cause death, or MACE (Figure 2). 
A

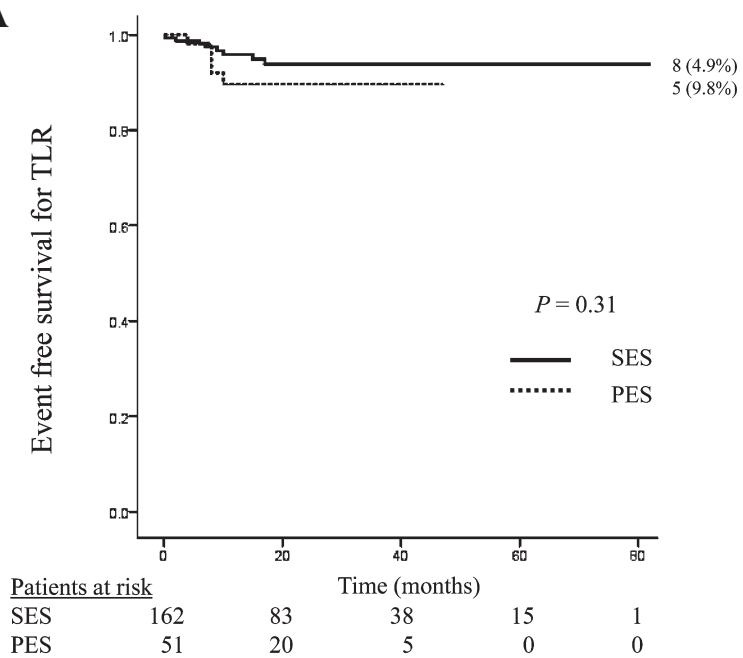

B

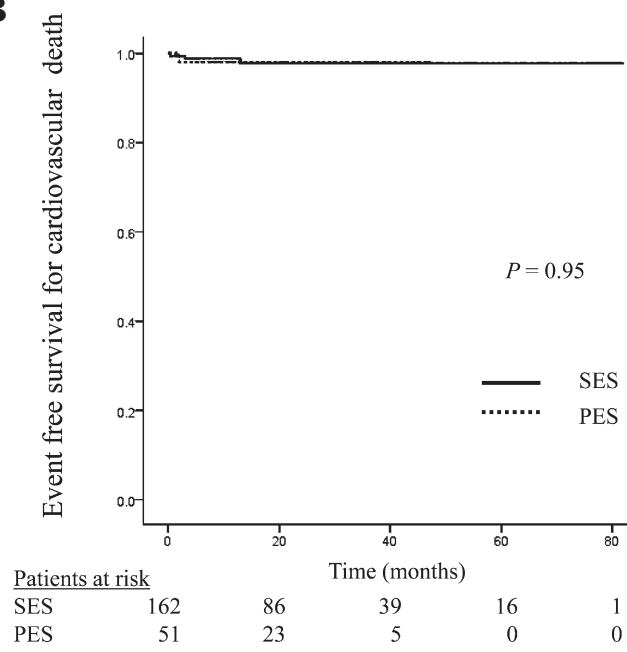

C

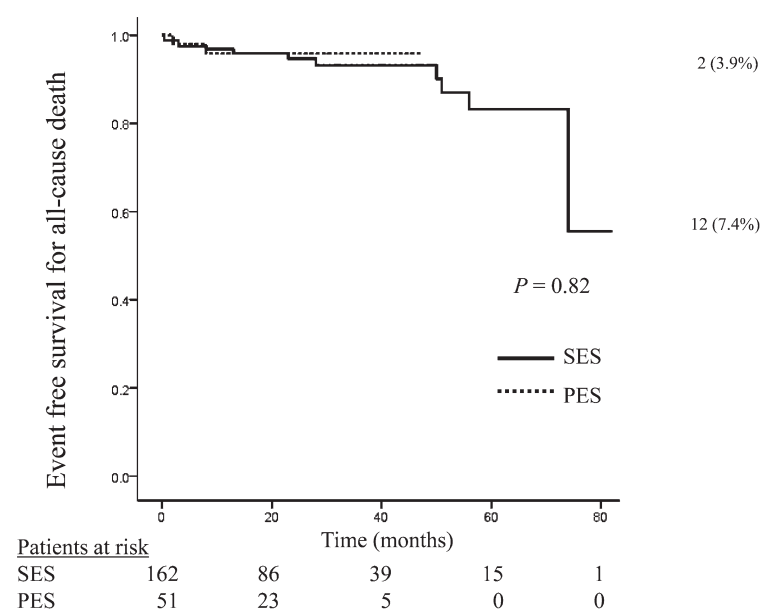

D

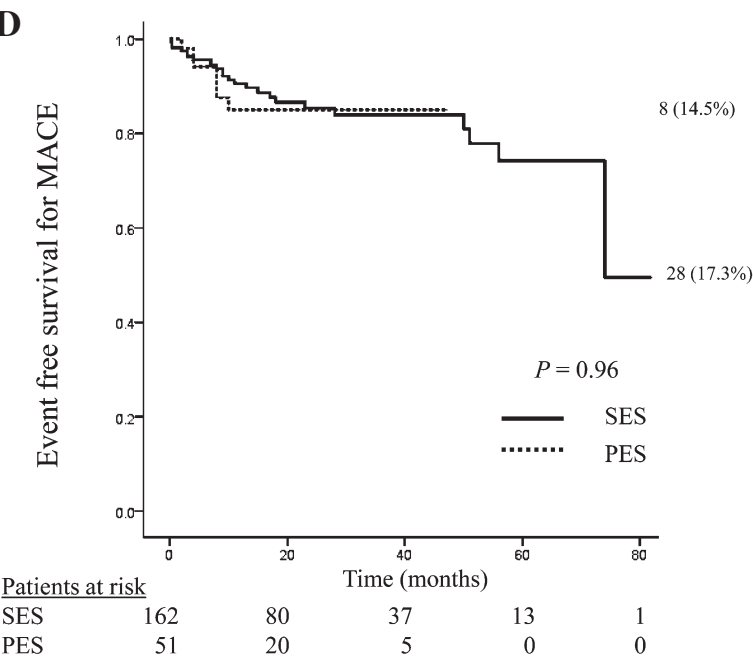

Figure 2. A: Kaplan-Meier curves for target lesion revascularization (TLR). B: Kaplan-Meier curves for cardiovascular death. C: Kaplan-Meier curves for all-cause death. D: Kaplan-Meier curves for major adverse cardiac events (MACE).

\section{Discussion}

The major findings of this study were that: 1) the longterm clinical outcomes were not significantly different between the SES group and PES group following RA; 2) the combined strategy of RA and DES for calcified or complex coronary lesions showed that the rate of revascularization was low during the long-term follow-up period.

Previous studies have compared clinical outcomes between SES and PES, and reported SES was superior in terms of risk reduction of re-intervention. ${ }^{19,20)}$ However, none of the studies reported significant differences with respect to death and myocardial infarction between SES and PES. Our study found no difference between the two stents with respect to the rate of MI, ST, and death during a median follow-up period of 21 months.

Several studies have showed that DES implantation after RA reduced the incidences of TLR $^{21)}$ and MACE. ${ }^{16,17)}$ Mezilis, et al reported that a combined strategy consisting of RA and
DES reduced the rate of MACE combined with TLR, TVR, stroke, MI, and occurrence of death in 150 patients with heavily calcified coronary artery lesions. They reported excellent TLR and MACE results of $2 \%$ and $11.3 \%$, respectively. The TLR and MACE rates were $6.1 \%$ and $14.6 \%$, respectively, which are higher than those of Mezilis, et al. However, the background characteristics of our patients and the complexities of the lesions, such as incidences of diabetes mellitus of $48.5 \%$, hemodialysis of $12.4 \%$, and chronic total occlusion of $12.4 \%$, seem to suggest our subjects could be categorized as extremely complex coronary lesion subsets. Furuichi, et al also reported the effectiveness of DES implantation following RA in 95 patients. In their study, the rates of TLR and MACE, defined as death, MI and TVR, were $9.5 \%$ and $15.8 \%$, respectively, which is comparable to our results. In these studies, multiple classes of DES were used, however, they did not analyze the differences among the classes. ${ }^{16,17)}$ The present study investigated the comparative clinical outcomes between SES and PES, and found no significant difference in the long-term 
clinical outcomes between the groups.

In the BMS era, the rates of TLR and restenosis after stent implantation following RA were high. Moussa, et al reported TLR and restenosis rates of $18.0 \%$ and $22.5 \%$, respectively. ${ }^{9)}$ Other studies also found high rates of TLR and restenosis after BMS implantation following RA. ${ }^{7,8)}$ The high rate of restenosis was thought to result from vessel injury and a neointimal response due to RA. ${ }^{22}$ DES might play a role of reducing the neointimal response to the injury through atherectomy. It is also speculated that lesion modification by RA could yield smooth stent delivery, prevent damage to the polymer coating, ${ }^{23)}$ and provide optimal stent expansion, all of which could help DES be effective for even calcified coronary lesions.

Study limitations: Our study was a single-center retrospective observational study with a relatively small number of subjects, which suggests a potential risk for bias and beta error. Furthermore, we may not be able to apply these results to current clinical practices where the new generation types of DES are widely used.

Conclusions: This study showed that there was no significant difference in the long-term clinical outcomes between SES and PES, both following RA.

\section{ACKNOWLEDGMENT}

Dr. Ako and Dr. Sakakura both received a speaking honorarium (minor) from Boston Scientific Corporation.

\section{REFERENCES}

1. Hoffmann R, Mintz GS, Popma JJ, et al. Treatment of calcified coronary lesions with Palmaz-Schatz stents. An intravascular ultrasound study. Eur Heart J 1998; 19: 1224-31.

2. Cutlip DE, Chauhan MS, Baim DS, et al. Clinical restenosis after coronary stenting: perspectives from multicenter clinical trials. J Am Coll Cardiol 2002; 40: 2082-9.

3. Fitzgerald PJ, Ports TA, Yock PG. Contribution of localized calcium deposits to dissection after angioplasty. An observational study using intravascular ultrasound. Circulation 1992; 86(1): 64-70.

4. Mintz GS, Potkin BN, Keren G, et al. Intravascular ultrasound evaluation of the effect of rotational atherectomy in obstructive atherosclerotic coronary artery disease. Circulation 1992; 86 1383-93.

5. Kovach JA, Mintz GS, Pichard AD, et al. Sequential intravascular ultrasound characterization of the mechanisms of rotational atherectomy and adjunct balloon angioplasty. J Am Coll Cardio 1993; 22: 1024-32

6. Ellis SG, Popma JJ, Buchbinder M, et al. Relation of clinical presentation, stenosis morphology, and operator technique to the procedural results of rotational atherectomy and rotational atherectomy-facilitated angioplasty. Circulation 1994; 89: 882-92.

7. Warth DC, Leon MB, O’Neill W, Zacca N, Polissar NL, Buch- binder M. Rotational atherectomy multicenter registry: acute results, complications and 6-month angiographic follow-up in 709 patients. J Am Coll Cardiol 1994; 24: 641-8.

8. Reifart N, Vandormael M, Krajcar M, et al. Randomized comparison of angioplasty of complex coronary lesions at a single center. Excimer Laser, Rotational Atherectomy, and Balloon Angioplasty Comparison (ERBAC) Study. Circulation 1997; 96: 91-8.

9. Moussa I, Di Mario C, Moses J, et al. Coronary stenting after rotational atherectomy in calcified and complex lesions. Angiographic and clinical follow-up results. Circulation 1997; 96: 12836.

10. Morice MC, Serruys PW, Sousa JE, et al. A randomized comparison of a sirolimus-eluting stent with a standard stent for coronary revascularization. N Engl J Med 2002; 346: 1773-80.

11. Stone GW, Ellis SG, Cox DA, et al. A polymer-based, paclitaxeleluting stent in patients with coronary artery disease. N Engl J Med 2004; 350: 221-31

12. Moses JW, Leon MB, Popma JJ, et al. Sirolimus-eluting stents versus standard stents in patients with stenosis in a native coronary artery. N Engl J Med 2003; 349: 1315-23.

13. Stone GW, Ellis SG, Cannon L, et al. Comparison of a polymerbased paclitaxel-eluting stent with a bare metal stent in patients with complex coronary artery disease: a randomized controlled trial. JAMA 2005; 294: 1215-23.

14. Khattab AA, Otto A, Hochadel M, Toelg R, Geist V, Richardt G. Drug-eluting stents versus bare metal stents following rotational atherectomy for heavily calcified coronary lesions: late angiographic and clinical follow-up results. J Interv Cardiol 2007; 20: $100-6$.

15. Clavijo LC, Steinberg DH, Torguson R, et al. Sirolimus-eluting stents and calcified coronary lesions: clinical outcomes of patients treated with and without rotational atherectomy. Catheter Cardiovasc Interv 2006; 68: 873-8.

16. Mezilis N, Dardas P, Ninios V, Tsikaderis D. Rotablation in the drug eluting era: immediate and long-term results from a single center experience. J Interv Cardiol 2010; 23: 249-53.

17. Furuichi S, Sangiorgi GM, Godino C, et al. Rotational atherectomy followed by drug-eluting stent implantation in calcified coronary lesions. EuroIntervention 2009; 5: 370-4.

18. Cutlip DE, Windecker S, Mehran R, et al. Clinical end points in coronary stent trials: a case for standardized definitions. Circulation 2007; 115: 2344-51.

19. Schömig A, Dibra A, Windecker S, et al. A meta-analysis of 16 randomized trials of sirolimus-eluting stents versus paclitaxeleluting stents in patients with coronary artery disease. J Am Coll Cardiol 2007; 50: 1373-80. (Review)

20. Windecker S, Remondino A, Eberli FR, et al. Sirolimus-eluting and paclitaxel-eluting stents for coronary revascularization. $\mathrm{N}$ Engl J Med 2005; 353: 653-62.

21. Kubota T, Ishikawa T, Nakano Y, et al. Retrospective comparison of clinical and angiographic outcomes after sirolimus-eluting and bare-metal stent implantation in 312 consecutive, nonrandomized severely calcified lesions using a rotablator. Int Heart J 2011; 52: 65-71.

22. Moussa I, Moses J, Colombo A. Atherectomy plus stenting: what do we gain? Semin Interv Cardiol 2000; 5: 217-25. (Review)

23. Kuriyama N, Kobayashi Y, Yamaguchi M, Shibata Y. Usefulness of rotational atherectomy in preventing polymer damage of everolimus-eluting stent in calcified coronary artery. JACC Cardiovasc Interv 2011; 4: 588-9. 